\title{
Anatomía Aplicada a La Cirugía de Los Tendones Extensores
}

\author{
M.R. Morro-Martí ${ }^{1,2} \quad$ M. Llusá-Pérez ${ }^{1,2}$ P. Forcada-Calvet ${ }^{3} \quad$ A. Carrera ${ }^{4} \quad$ A. Mustafa-Gondolbeu ${ }^{1,2,3}$ \\ ${ }^{1}$ Departamento de Anatomía y Embriología Humana, Laboratorio de \\ Macro-Micro Disección y Anatomía Quirúrgica, Facultad de \\ Medicina, Universidad de Barcelona, Barcelona, Spain \\ 2 Servicio COT, Hospital Clínic de Barcelona, Barcelona, Barcelona, Spain \\ ${ }^{3}$ Servicio COT, Hospital Universitario Arnau de Vilanova, Lleida, Spain \\ ${ }^{4}$ Unidad de Anatomía, Facultad de Medicina, Universidad de Girona, \\ Girona, Spain \\ Rev Iberam Cir Mano 2019;47:56-64.

\begin{abstract}
Address for correspondence Manuel Llusá Pérez, MD, PhD, Departamento de Anatomía y Embriología Humana, Facultad de Medicina, Universidad de Barcelona, C/ Casanova, Barcelona,
\end{abstract} \\ 143. 08036, Spain (e-mail: mllusa@ub.edu).
}

\section{Resumen \\ Palabras Clave \\ - anatomía \\ - tendón \\ - extensores \\ - dedo en martillo \\ - dedo en hojal}

\section{Abstract}

DManuel Llusá Pérez's ORCID is https://orcid.org/0000-0003$3265-2778$

received

April 11, 2019

accepted

April 16, 2019
La anatomía de los tendones extensores de los dedos es muy variable en el dorso de la mano y la formación del aparato extensor en los dedos, junto con la musculatura intrínseca de la mano, es muy compleja. Su conocimiento preciso es imprescindible para la comprensión de la fisiopatología de las lesiones de esas estructuras y su tratamiento. Además, se tratan de lesiones muy frecuentes debido a su localización subcutánea en todo su recorrido, pero especialmente en los dedos.

En el dorso de la muñeca, los tendones extensores se distribuyen en seis correderas osteofibrosas muy bien delimitadas.

En los dedos, los tendones extensores extrínsecos se unen con la musculatura intrínseca de la mano (músculos interóseos y lumbricales), para formar el complejo aparato extensor. Sus componentes principales son la bandeleta central encargada de la extensión IFP y las bandeletas laterales que se acaban uniendo formando en tendón terminal responsable de la extensión IFD. Además, se encuentran unas fibras de conexión en las articulaciones MCF e IFP, las bandeletas sagitales y los ligamentos retinaculares transversos respectivamente, que unen el aparato extensor a la cara anterior del dedo manteniéndolo en eje sobre la cara convexa de las articulaciones.

The anatomy of the extensor tendons of the fingers is very variable at the dorsum of the hand and the extensor apparatus of the fingers is very complex. Its precise knowledge is critical for the understanding of the physiopathology of their lesions and their treatment. Besides, lesions of the extensor tendons are frequent as they are subcutaneous in all their way, but specially at the fingers.

At the dorsum of the wrist, the extensor tendons are distributed in six extensor compartments.

At the fingers, the extrinsic extensor tendons find the intrinsic muscles of the hand (interossei and lumbricals) to form the complex extensor apparatus. Its main 
components are the central slip responsible for the extension of the PIP joint and the lateral bands that finally joint to form the terminal tendon responsible for the extension of the DIP joint. Besides, there are some connection fibres in the MCP and PIP joints, the sagittal bands and the transverse retinacular ligaments respectively, which connect the extensor apparatus to the anterior region of the finger, maintaining it in line with the finger axis over the convex side of the joints.

\section{Introducción}

Las lesiones de los tendones extensores a nivel de la muñeca, mano y dedos, son frecuentes debido a su localización superficial y exposición a agentes traumáticos. Se pueden producir lesiones por pequeñas heridas cutáneas o por grandes traumatismos complejos con pérdida de partes blandas y fracturas asociadas. Las rupturas tendinosas subcutáneas suelen pasar desapercibidas inicialmente y pueden llegar a ser desconcertantes.

Es esencial un conocimiento anatomofisiológico amplio para poder diagnosticar de una manera precisa esas lesiones, así como su repercusión funcional inmediata y tardía. Hay que conocer los mecanismos patogénicos de las deformidades digitales para poder comprender como se instauran, así como su diagnóstico y tratamiento.

\section{Anatomía de Los Tendones Extensores}

En el dorso de la muñeca los tendones extensores se disponen bajo el retináculo extensor que delimita seis compartimentos para su paso hacia la mano (-Fig. 1). Dentro de cada compartimento o corredera osteofibrosa podemos encontrar subdivisiones en caso de contener más de un tendón. ${ }^{1}$

La primera corredera, sobre la estiloides radial, aloja a los tendones del abductor pollicis longus (APL) y extensor pollicis brevis (EPB), frecuentemente en un subcompartimento no siempre constante.

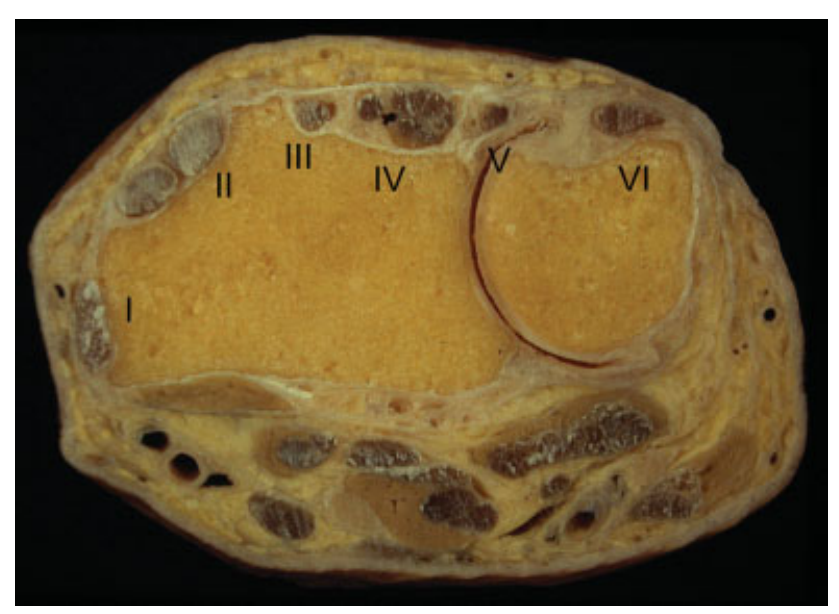

Fig. 1 Corte transversal a nivel de la muñeca mostrando la disposición de los compartimentos osteofibrosos y sus tendones. I: APL y EPB; II: ECRL y ECRB; III: EPL; IV: ED y EIP; V: EDM; VI: ECU.
La segunda corredera contiene los tendones del extensor carpi radialis longus (ECRL) y brevis (ECRB). Su localización en relación al tendón del extensor pollicis longus (EPL), que discurre por la tercera corredera, varía si la posición de la mano está en supinación (descripción clásica) o en pronación. El EPL se refleja hasta $40^{\circ}$ sobre el tubérculo dorsal del radio o tubérculo de Lister, que forma el límite externo de la tercera corredera.

La cuarta corredera contiene los tendones del extensor digitorum (ED) (3 o 4 según exista o no tendón para el quinto dedo a ese nivel), y el tendón del extensor indicis (EI) o extensor indicis proprius (EIP), que se localiza por debajo del común. Hay que mencionar que en el lado más radial de esa corredera podemos encontrar a nivel del suelo óseo, la rama terminal del nervio interóseo posterior acompañado por un pequeño paquete vascular formado per la rama dorsal de la anteria interósea anterior, junto con sus venas concomitantes.

La quinta corredera aloja el tendón del extensor digiti minimi (EDM) pudiendo encontrar uno o dos tendones. El suelo de esa corredera se sitúa en el intersticio existente entre los extremos distales del radio y cúbito. En el fondo de ese compartimento, se localiza el segmento distal de la arteria interósea posterior, que se anastomosa con el arco transverso dorsal del carpo y que en esa zona también se describe con el nombre de arteria del quinto compartimento extensor. Antes de introducirse en esa corredera, la arteria interósea posterior forma un arco que se comunica con la rama dorsal de la arteria interósea anterior.

La sexta corredera aloja el tendón del extensor carpi ulnaris (ECU). Su constitución es más compleja definiéndose dos capas. La hoja profunda forma parte de la vaina del ECU originándose en el borde externo del surco dorsal de la cabeza del cúbito, cubriendo en tendón del ECU e insertándose en el borde interno del mismo surco. La hoja superficial (el propio retináculo extensor), forma una estructura a modo de cincha alrededor del tendón siendo un área independiente que permite los cambios de posición del tendón durante la pronosupinación.

En el dorso de la mano, los tendones extensores se encuentran por debajo de la aponeurosis superficial de la mano (continuación distal del retináculo extensor). Su disposición y número es variable (-Fig. 2) ${ }^{2,3}$

Los tendones de los dedos medio y anular se encuentran alineados con el eje del metacarpiano correspondiente, mientras que los tendones del índice y meñique cruzan oblicuamente para llegar a buscar el aparato extensor correspondiente. Los tendones del ED se encuentran conectados por unas láminas 


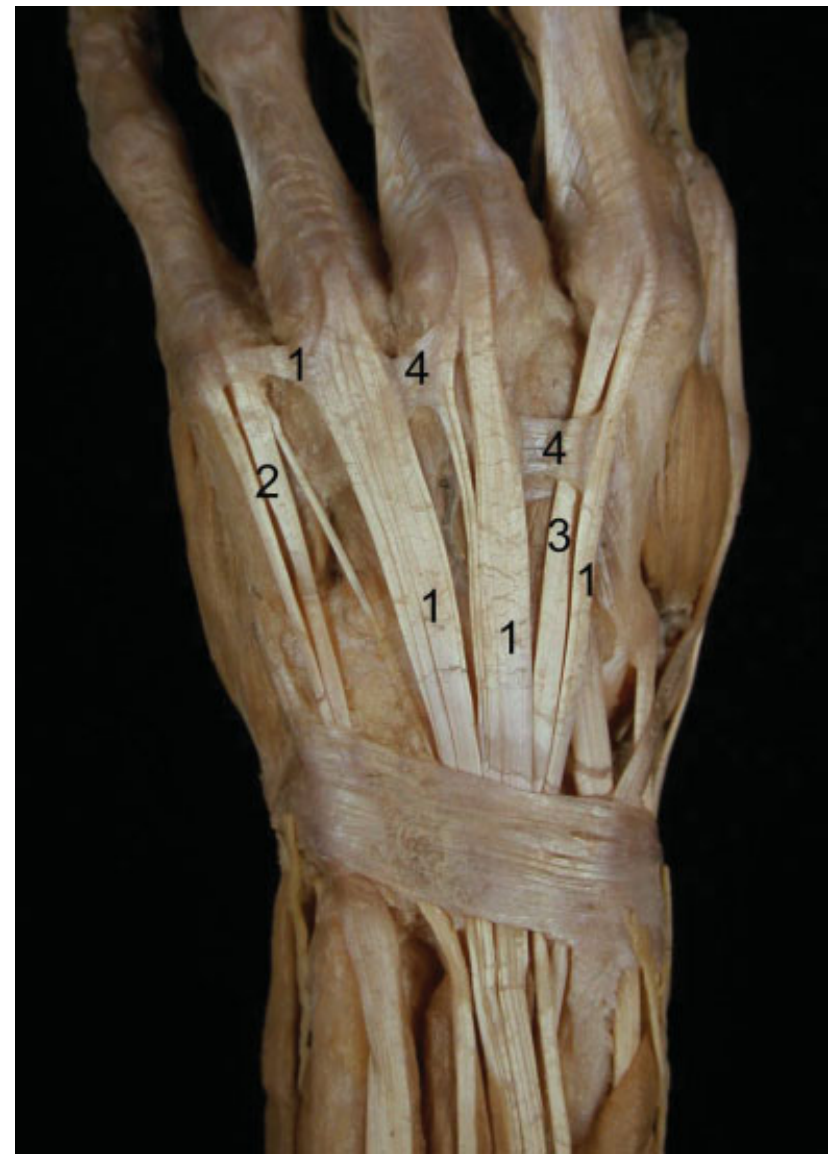

Fig. 2 Disposición de los tendones extensores en el dorso de la muñeca y mano. 1: ED; 2: EDM; 3: EIP; 4: juncturae tendinum. Obsérvese la presencia de pequeños tendones supranumerarios.

aponeuróticas, más o menos engrosadas, e incluso por verdaderas conexiones de tipo tendinoso, denominadas connexus intertendineus o juncturae tendinum.

El número de tendones para cada dedo puede ser variable, pudiendo apreciarse, especialmente en el tercer y cuarto dedos, varios constituyentes tendinosos destinados a un mismo dedo.

Podemos concretar que el índice presenta un tendón proviniente del ED que se localiza más radial y superficial que el extensor propio que se localiza más cubital y profundo. Las fibras aponeuróticas que unen el borde cubital del tendón común del índice con el dedo medio son el mejor reparo para distinguir el tendón común del propio. Con frecuencia podemos encontrar variaciones anatómicas con un tendón doble del EI situado uno a cada lado del común.

Respecto a los tendones del tercer y cuarto dedos, no es excepcional encontrar, además de los del extensor común, variaciones anatómicas como pueden ser tendones del músculo manio o extensor corto de los dedos.

El meñique es un caso especial. En primer lugar, no hay que confundir el componente del ED para el quinto dedo con una juncturae tendinum, ni pensar si existen dos tendones del extensor propio, en que uno de ellos pueda ser el común. Las variaciones son frecuentes. $^{2,3}$ También hay que señalar la existencia de la formación de compartimentos osteofibrosis para esos tendones en el dorso de la mano.
La inserción de los tendones extensores se realiza en la base de la falange proximal con expansiones a la cápsula posterior MTCF. A partir de ahí, sus fibras forman parte del complejo aparato extensor de los dedos.

En el pulgar, hay que mencionar que el tendón del APL presenta múltiples fascículos tendinosos con subdivisiones que también pueden confundirnos. Es de destacar el grosor de ese tendón terminal y la inserción en la base del primer metacarpiano con expansiones al trapecio y a la musculatura de la eminencia tenar.

El tendón del EPB es variable en grosor pudiendo incluso estar ausente o duplicado. Su inserción se realiza en la base de la primera falange confundiéndose con el aparato extensor.

El tendón del EPL es grueso y se caracteriza por la gran reflexión que efectúa sobre el tubérculo dorsal del radio para ir a buscar su inserción en la base de la falange distal y aparato extensor del pulgar. Comentamos que la relación de los tendones que forman los límites de la tabaquera anatómica es variable respecto a los tendones del ECRL y ECRB según se encuentre la mano en pronación o supinación. En ocasiones puede estar duplicado.

La cadena articular de los dedos está controlada por los tendones extrínsecos, flexores y extensores existiendo además sistemas laterales formados por los músculos intrínsecos que regularán con precisión, a través del aparato extensor, los movimientos que dependerán del sistema extrínseco. El desequilibrio de ese balance entre extrínsecos e intrínsecos, conlleva la disfunción de la mano con deformidades diversas dependiendo del mecanismo patogénico. ${ }^{4}$

\section{Anatomía del Aparato Extensor}

En la cara dorsal de los dedos trifalángicos, se constituye un complejo sistema de fibras tendinoaponeuróticas entrecruzadas que por su función principal es conocido como Aparato Extensor de los Dedos ${ }^{5-7}$ (-Fig. 3).

En la formación del aparato extensor participan tendones extensores extrínsecos, es decir, procedentes de músculos extensores originados en el antebrazo, y tendones de músculos intrínsecos de la mano.

En la cara dorsal de la articulación MTCF de cada dedo trifalángico llega un tendón del músculo extensor común de

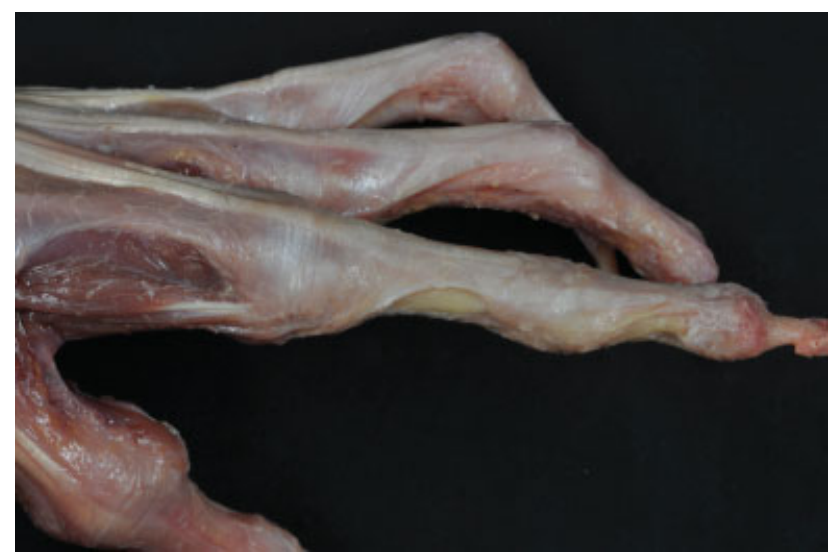

Fig. 3 Visión lateral del aparato extensor in situ en 3 dedos. 
los dedos y en el caso de los dedos índice y meñique llega también el tendón de su músculo extensor propio (siempre en una disposición cubital en relación al tendón del extensor común). En ese punto, esos tendones extensores extrínsecos forman el denominado "extensor largo" sobre el cual se centra la formación de todo el aparato extensor (-Fig. 4a y fig. 4b). Para comprender su descripción es necesario identificar cada uno de sus elementos en los esquemas (-Fig. 4c).

Durante su paso sobre la articulación MTCF, el extensor largo desprende a ambos lados las denominadas bandeletas o cintillas sagitales que, abrazando la articulación, van a insertarse en su placa palmar $\mathrm{y}$ en el ligamento intermetacarpiano transverso. Esas bandeletas mantienen al tendón extensor en eje sobre la superficie dorsal convexa de la cabeza metacarpiana en el transcurso de la flexión de la articulación, evitando su luxación en los "valles» intermetacarpianos (-Fig. 5a y fig. 5b). Un poco más distalmente, el extensor largo emite una expansión profunda (-Fig. 6), que puede ser más o menos robusta y que, confundiéndose con la cápsula articular, va a insertarse en la base de la primera falange. A través de esas inserciones profundas y laterales, el extensor largo puede realizar la extensión de la primera falange del dedo.

A partir de la articulación MTCF, el tendón del extensor largo se divide en dos cintillas laterales y una cintilla central o media (-Fig. 4a y fig. 4b). A esas divisiones se van a unir fibras procedentes de los músculos intrínsecos de la mano: músculos interóseos y músculos lumbricales.
Los tendones de los músculos interóseos se dirigen a la región dorsal del dedo pasando dorsalmente respecto al ligamento intermetacarpiano transverso. Se incorporan al aparato extensor pasada la articulación MTCF pero antes realizan una primera inserción a nivel de los tubérculos laterales de la base de la primera falange y en la cápsula articular de la MTCF, a través de las cuales llevan a cabo las funciones de abducción si se trata de un interóseo dorsal o de adducción si se trata de un interóseo ventral (-Fig. 5a). Una vez realizada esa inserción, el tendón interóseo emite distintos tipos de fibras que se incorporan al propio aparato extensor (-Fig. 4a, fig.4b y fig. 5a):

- unas fibras transversales, arqueadas dorsalmente cubriendo la articulación MTCF y la base de la falange proximal, que llegan a fusionarse con las fibras transversales las cuales proceden del interóseo del lado opuesto, constituyendo así el llamado ligamento en dosel o dosel de los interóseos. A través de la formación de esa estructura, los interóseos ejercen una acción de flexión de la articulación MTCF.

- unas fibras oblicuas o cintilla medial, que se une a la cintilla central del tendón del extensor largo.

- unas cintillas laterales que van a fusionarse con la cintilla lateral del tendón del extensor largo. En las cintillas laterales del lado radial de cada dedo, se incorporan también el tendón de un músculo lumbrical. Los músculos lumbricales, a diferencia de los músculos interóseos, llegan al aparato extensor pasando ventralmente respecto al ligamento intermetacarpiano transverso.

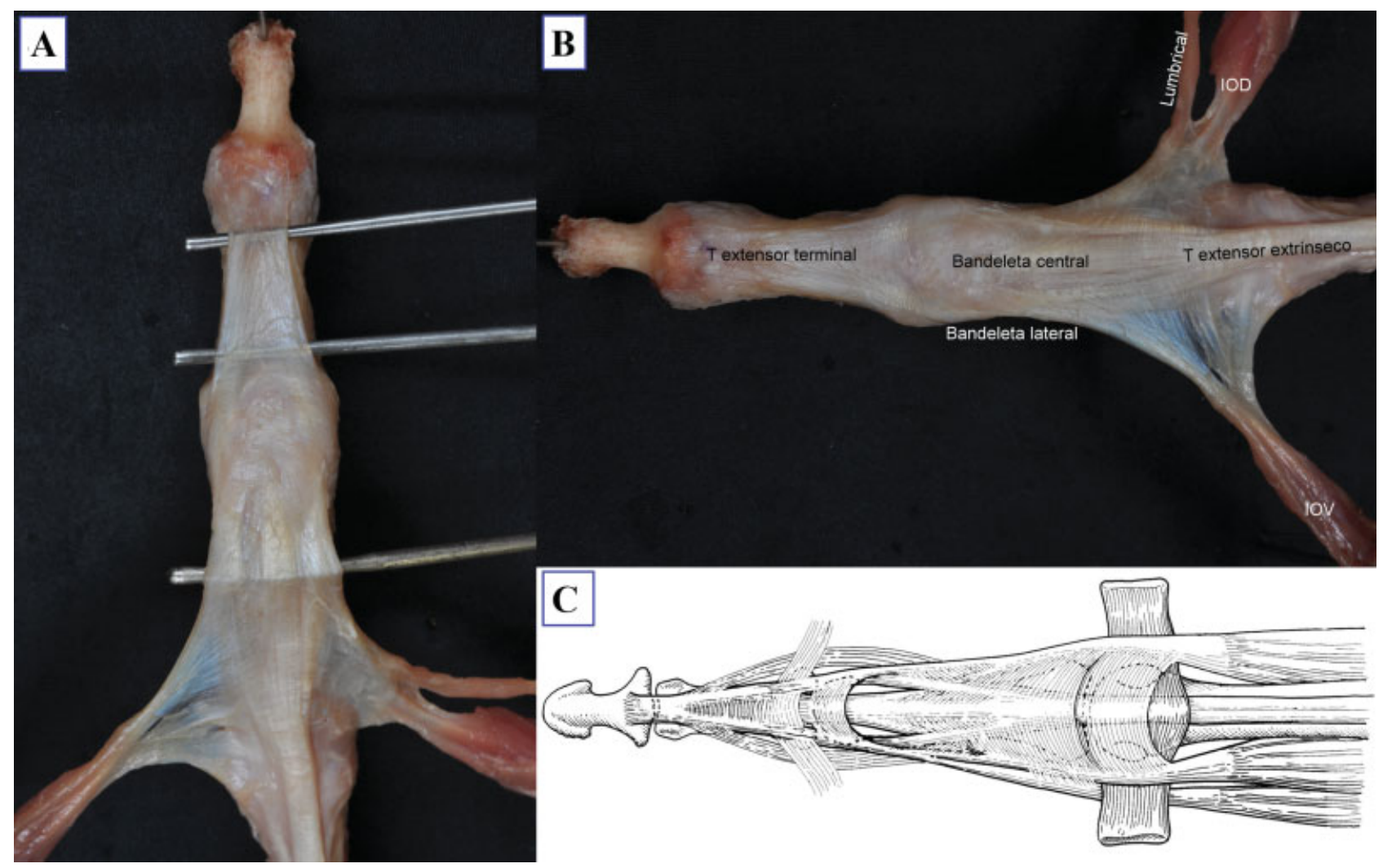

Fig. 4 (A) Visión dorsal del aparato extensor tensando sus diferentes componentes con unas agujas de Kirschner. (B) Visión dorsal del aparato extensor de los dedos con sus diferentes componentes. (C) Esquema de una visión dorsal del aparato extensor. 

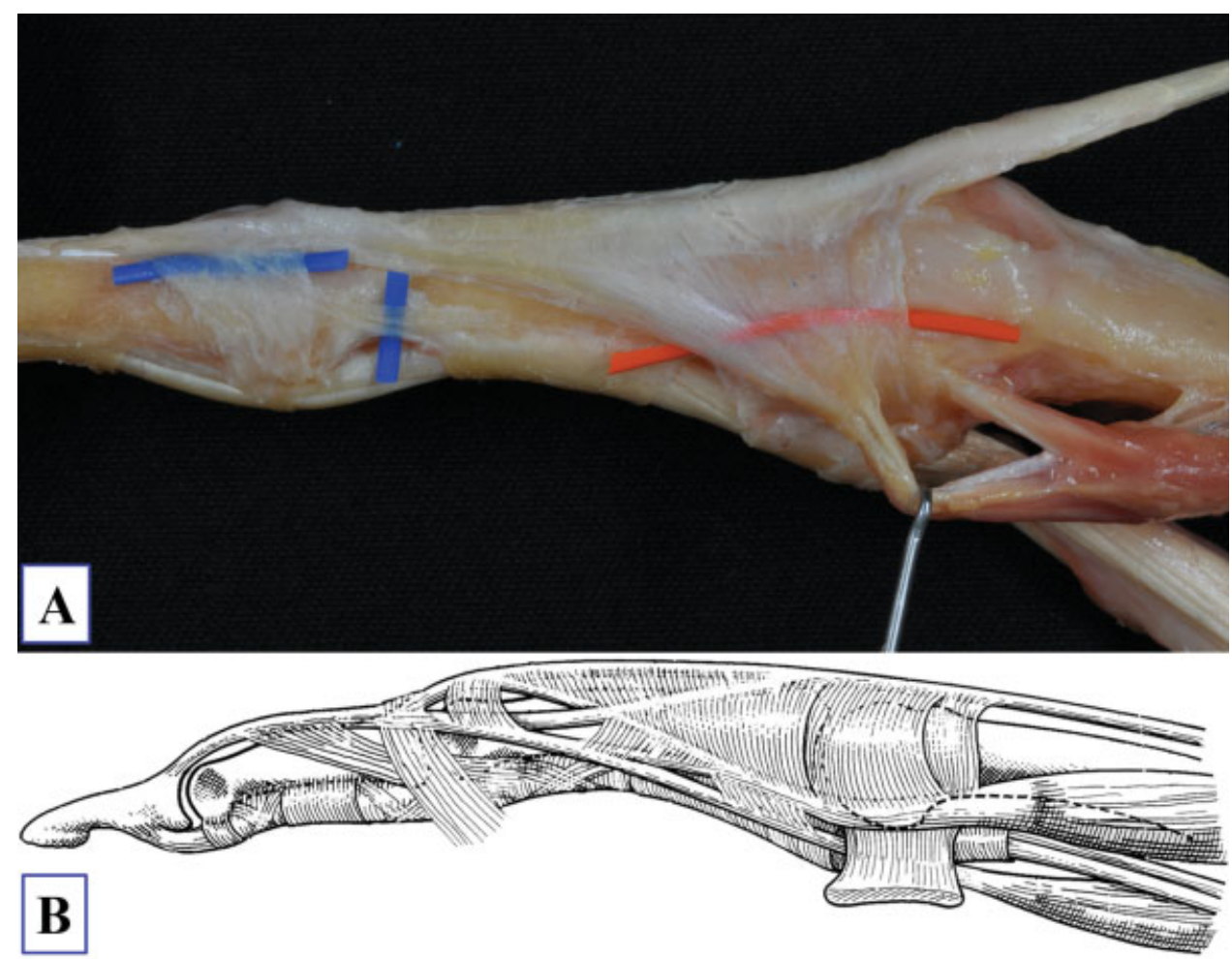

Fig. 5 (A) Visión lateral del aparato extensor. El vesseloop rojo destaca los elementos de conexión MCF (la bandeleta sagital) y los azules los elementos de conexión IFP (ligamento retinacular transverso y oblícuo de Landsmeer). (B) Esquema de una visión lateral del aparato extensor.

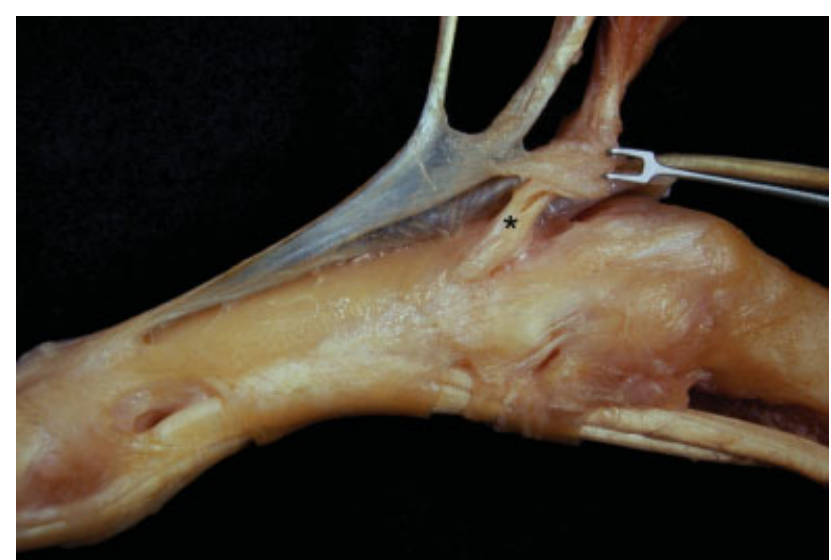

Fig. 6 Visión lateral del aparato extensor pudiendo apreciar la expansión profunda del ED a nivel de la base de la falange proximal (*).

La unión de la cintilla central del extensor largo con las cintillas mediales de los músculos interóseos, forma el tendón medio del aparato extensor que llega a insertarse en el tubérculo dorsal la base de la falange media, y a través del cual se ejerce la extensión de la articulación IFP.

Por otro lado, la unión de las cintillas laterales (del extensor largo y de los interóseos), forma la bandeleta lateral conjunta o tendón extensor lateral ( - Fig. 4a y fig. 4b). Los dos tendones extensores laterales inicialmente situados en posición dorsolateral, a ambos lados de la articulación IFP continúan su trayecto distal y una vez pasada la articulación inician un entrecruzamiento de fibras conocido como ligamento triangular hasta acabar completamente unidos a nivel del tercio distal de la falange media constituyendo el tendón terminal ( - Fig. 4a y fig. 4b) del aparto extensor que realiza su inserción en la falange distal y es, por tanto, el responsable de la extensión de la articulación IFD.

Durante el paso de los tendones extensores laterales por la cara dorsolateral de la IFP, cada uno emite lateralmente unas fibras transversas conocidas como ligamento retinacular transverso de Landsmeer que se inserta lateralmente sobre la placa volar (-Fig. 5A y B). Los ligamentos retinaculares transversos impiden que esos tendones extensores laterales se desplacen hacia el dorso del dedo durante la extensión de la articulación IFP (actúan de forma similar a las bandeletas sagitales a nivel de la articulación MTCF).

Existe también un ligamento retinacular oblícuo de Landsmeer, de difícil visualización, e inconstante, que proviene de la zona proximal de la vaina fibrosa de los flexores y tomando una dirección dorsal y distal, cruza oblicuamente la articulación IFP, entre el ligamento colateral y el ligamento retinacular transverso, hasta unirse a los laterales del complejo extensor a lo largo de la región de la falange media ( - Fig. 5A y B). Los ligamentos retinaculares oblicuos, pasan por delante del eje de flexión de la IFP y por detrás del eje de flexión de la IFD y se tensan durante los movimientos de flexión de la articulación IFD, conllevando una flexión automática de la IFP; y viceversa, la extensión de la IFP conlleva una flexión automática de la IFD ( - Fig. 7). De esa manera, desempeñan una función de coordinación de los movimientos de flexoextensión, solidarizando las dos articulaciones interfalángicas. $^{6,7}$ 


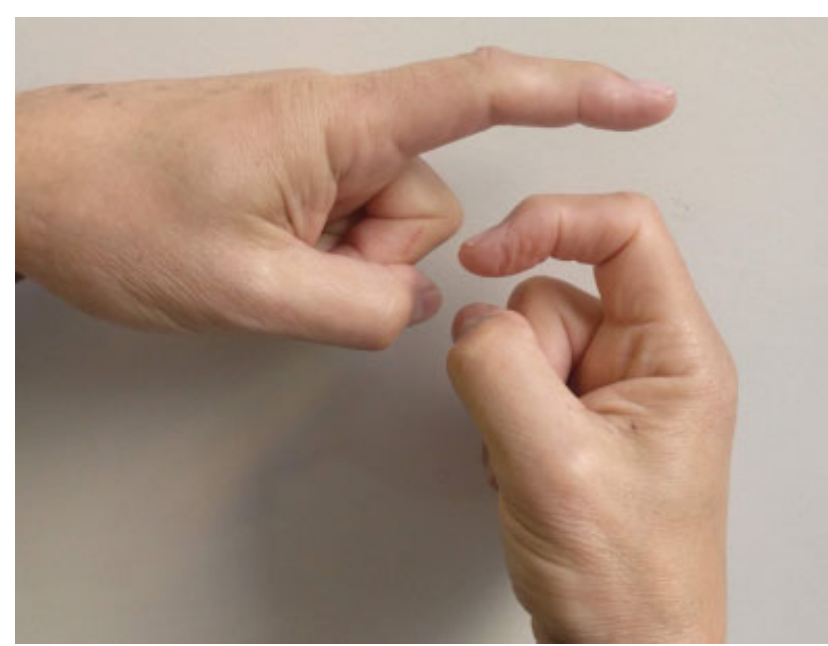

Fig. 7 Dedo índice izquierdo en extensión de las articulaciones IFP e IFD por acción de los ligamentos retinaculares oblícuos de Landsmeer: Dedo índice derecho en flexión de las articulaciones IFP e IFD también por la acción de los ligamentos retinaculars oblícuos de Landsmeer.

En el pulgar, la constitución del aparato extensor varía sustancialmente de la de los dedos trifalángicos. La contribución extrínseca proviene del extensor pollicis brecis (EPB), cuando está presente y extensor pollicis longus (EPL) y la contribución intrínseca le llega a través del abductor pollicis brevis (APB) por el lado radial y el adductor pollicis por el lado cubital (-Fig. 8a, fig.8b y fig. 8c). Las dos contribuciones se unen a nivel MTCF a través de unas fibras transversales que estabilizan el EPL sobre la cabeza del metacarpiano. El EPB se inserta en la base de la falange proximal aunque sólo en un $20 \%$ de los casos presenta inserciones óseas. El EPL, localizado cubitalmente respecto al tendón del EPB, se continúa distalmente hasta insertarse en la base de la falange distal. Pasada la articulación IF, ese tendón se aplana para formar el verdadero aparato extensor del pulgar al recibir las aportaciones intrínsecas del APB y adductor pollicis. Cabe destacar que la articulación MTCF del pulgar se comporta como la IFP de un dedo trifalángico y la IF es equivalente a la articulación IFP de un dedo largo.

\section{Lesiones Tendinosas y Exploración Física}

Kleinert y Verdan en 1983 propusieron un sistema de clasificación de las lesiones tendinosas de la mano, muñeca y antebrazo describiendo ocho zonas. Posteriormente, Doyle sugirió añadir una novena zona para las lesiones de los músculos extensores del tercio medio y proximal del antebrazo. La Federación Internacional de Socidedades de Cirugía de la Mano (IFSSH) considera estas zonas ( - Fig. 9): ${ }^{8,9}$

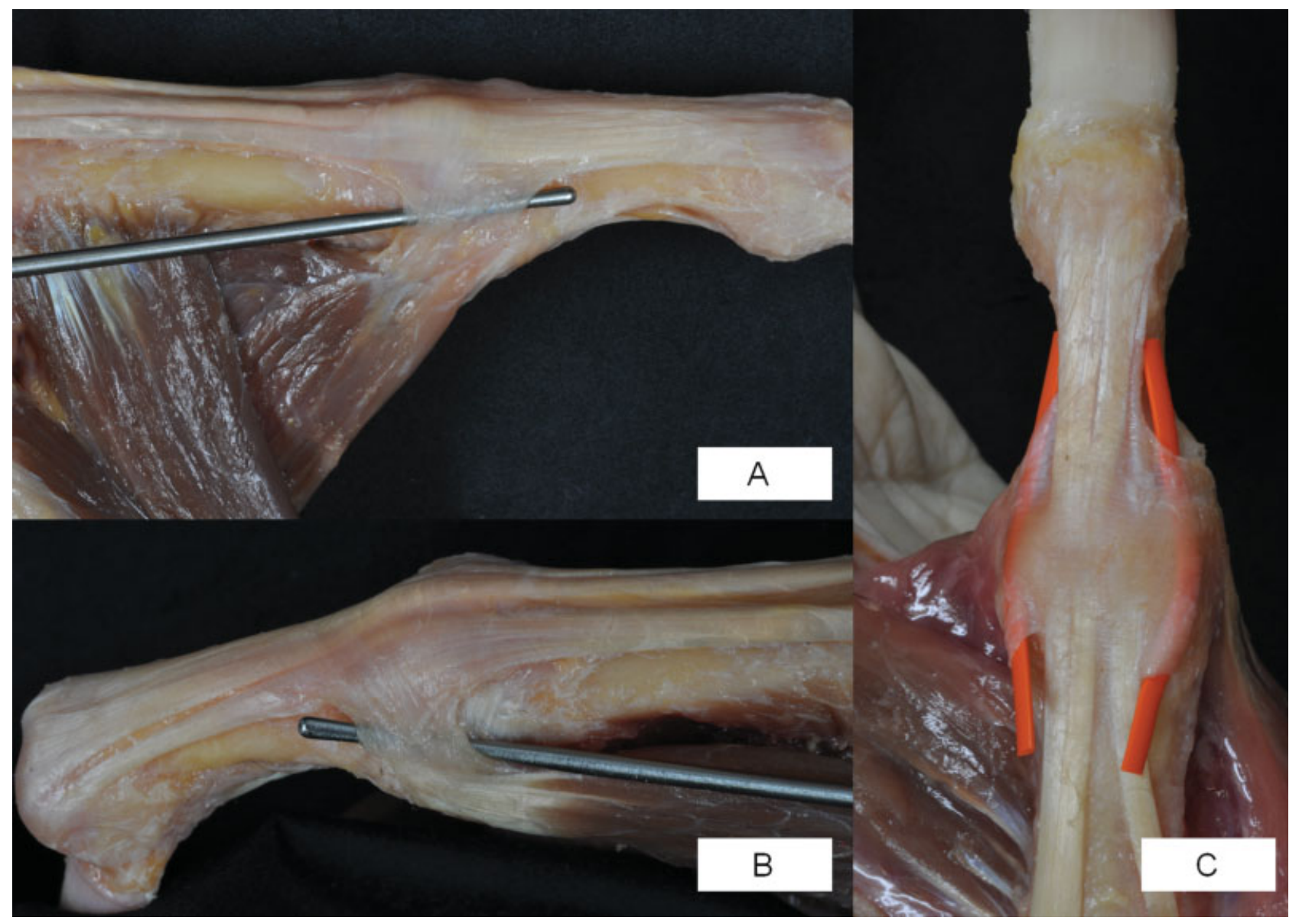

Fig. 8 (A) Visión lateral cubital del pulgar donde se observa la incorporación del tendón del adductor pollicis al aparto extensor. (B) Visión lateral radial del pulgar donde se ve la contribución del APB al aparato extensor. (C) Visión dorsal del pulgar destacando con dos vesseloops rojos las dos contribución intrínsecas, radial y cubital, al aparato extensor. 


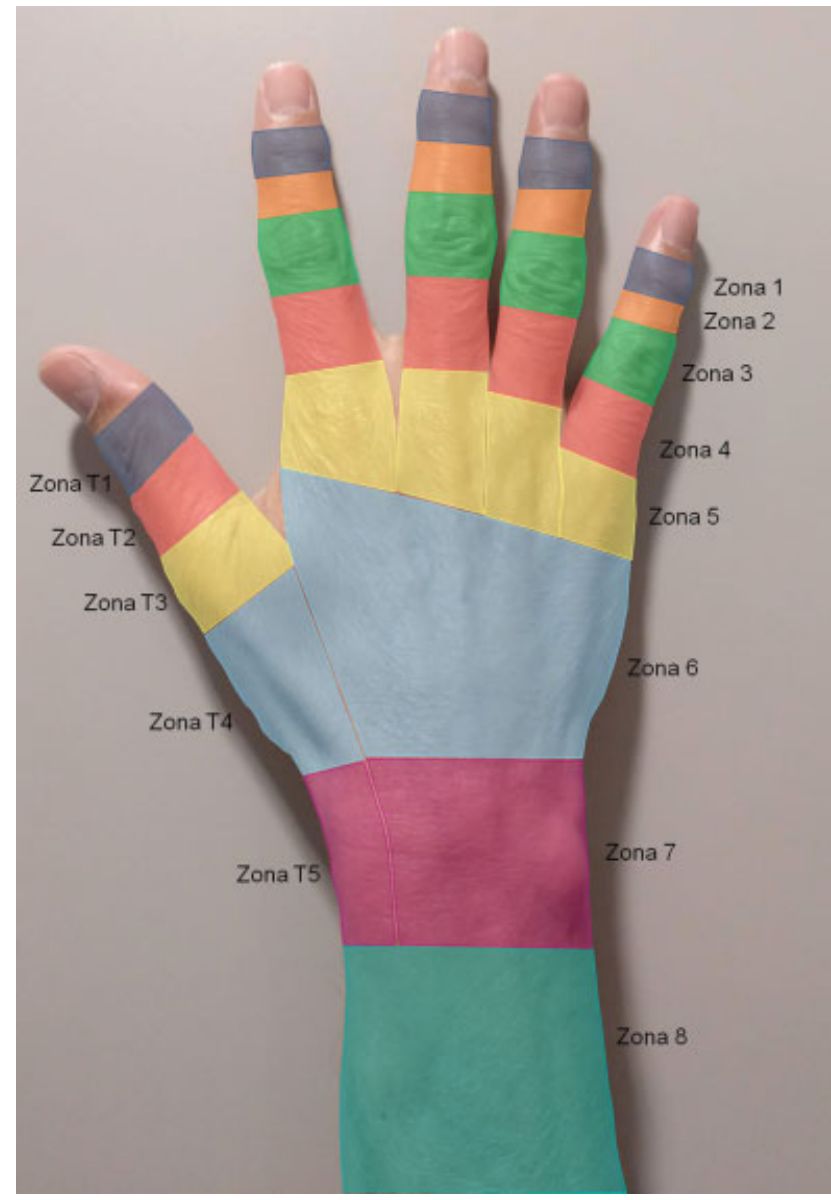

Fig. 9 Zonas de lesión de los tendones extensores de los dedos trifalángicos y del pulgar según la Federación Internacional de Sociedades de Cirugía de la Mano.

- Zona 1: Articulación interfalángica distal.

- Zona 2: Falange media.

- Zona 3: Articulación interfalángica proximal.

- Zona 4: Falange proximal.

- Zona 5: Articulación metacarpofalángica.

- Zona 6: Metacarpiano.

- Zona 7: Bajo el retináculo extensor (zona del radio distal).

- Zona 8: Tercio distal del antebrazo (unión miotendinosa).

- Zona 9: Tercio medio y proximal del antebrazo (masa muscular extensora).

A nivel del pulgar, la nomenclatura añade una $\mathrm{T}$ (thumb en inglés) delante del número de la zona. Las zonas descritas son:

- Zona T1: Articulación interfalángica.

- Zona T2: Falange proximal.

- Zona T3: Articulación metacarpofalángica.

- Zona T4: Metacarpiano.

- Zona T5: Bajo el retinánculo extensor (zona del radio distal).

A continuación vamos a detallar algunas particularidades clínicas y anatómicas y biomecánicas de algunas de esas zonas:

Zona 1: Dedo en martillo (-Fig. 10)

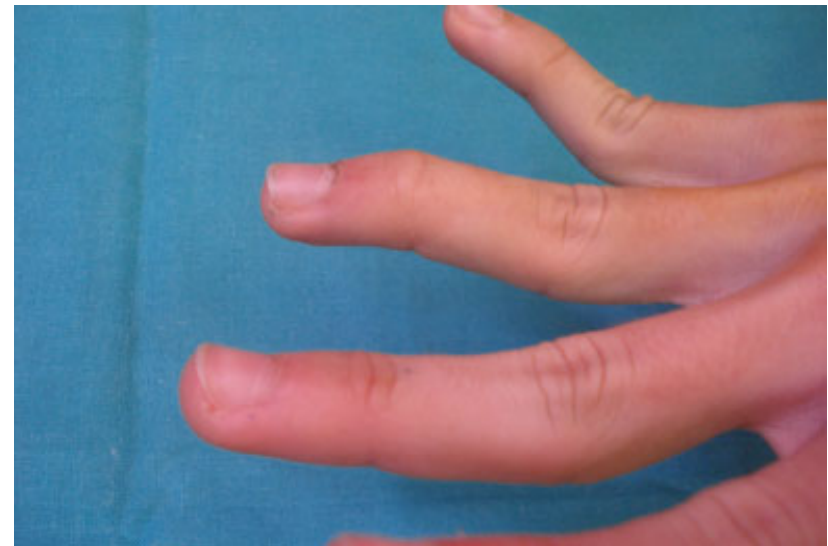

Fig. 10 Imagen clínica de un dedo anular en martillo por lesión del tendón terminal en la articulación IFD que secundariamente desarrolla una deformidad en cuello de cisne por hiperlaxitud (visible también en el dedo meñique, este caso sin lesión tendinosa).

La lesión del tendón terminal produce un déficit de extensión de la IFD de forma aguda, asociada a un pequeño fragmento óseo de la base de la F3 o no. En personas laxas, la tracción proximal del aparato extensor puede producir una hiperextensión de la interfalángica proximal desarrollando un dedo en cuello de cisne secundario.

\section{Zona 3: Dedo en ojal o en Boutonnière (-Fig. 11)}

La sección de la bandeleta central del aparto extensor, inicialmente no suele dar una deformidad en flexión de la IFP sino que además de permite en mayor o menor grado la extensión de la IFP. Progresivamente, se va produciendo el ojal en el aparato extensor sobre la IFP y las bandeletas laterales se van desplazando ventralmente hasta disponerse en un aposción ventral al eje de flexión de la IFP que provoca esa deformidad. Secundariamente, el aparato extensor produce la hiperextensión de la IFD inicialmente reductible, y con el tiempo, fija.

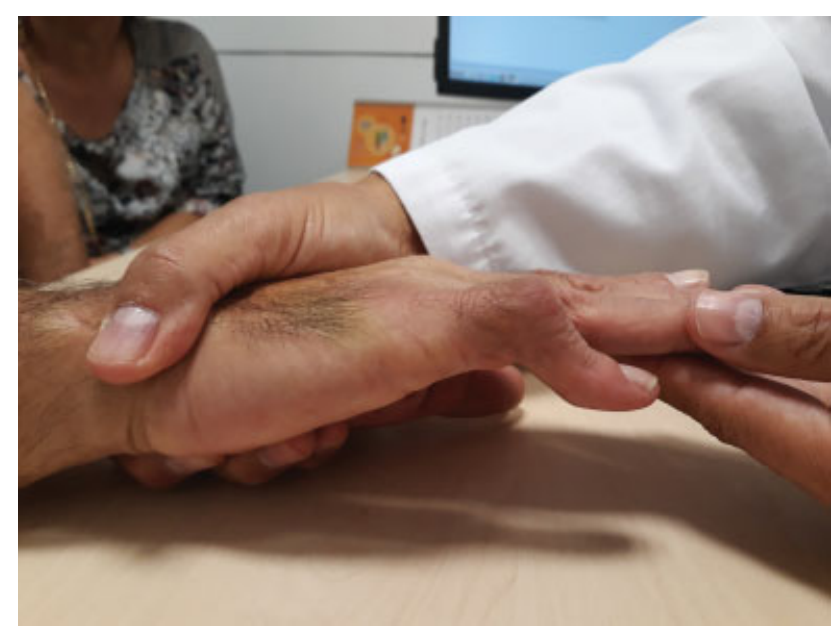

Fig. 11 Imagen clínica de un dedo en ojal o Boutonnière, con flexión de la articulación IFP e hiperextensión de la IFD. 


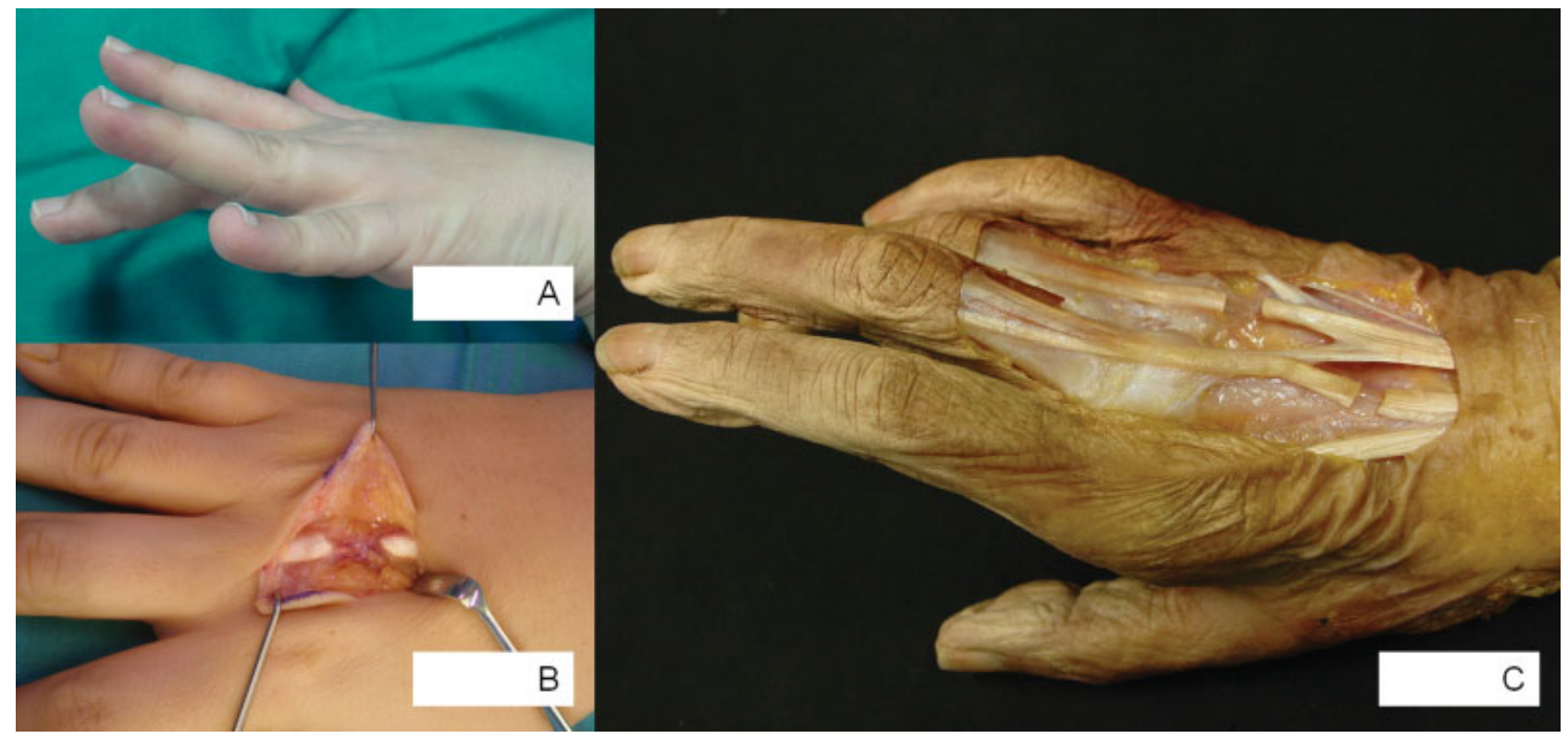

Fig. 12 (A) Imagen clínica de una sección del tendón extensor del dedo medio proximal a la juncturae tendinum al cabo de dos semanas de la lesión donde ya hay un déficit de extensión del dedo afecto. (B) Imagen intraoperatoria donde se observa la sección completa del tendón extensor. (C) Disección anatómica de los tendones extensores de la mano donde se ha reproducido una sección tendinosa proximal a la juncturae tendinum en el dedo medio observándose la conservación de la capacidad extensora del dedo; y una sección tendinosa distal a la juncturae tendinum donde ya existe un déficit de extensión desde el primer momento.

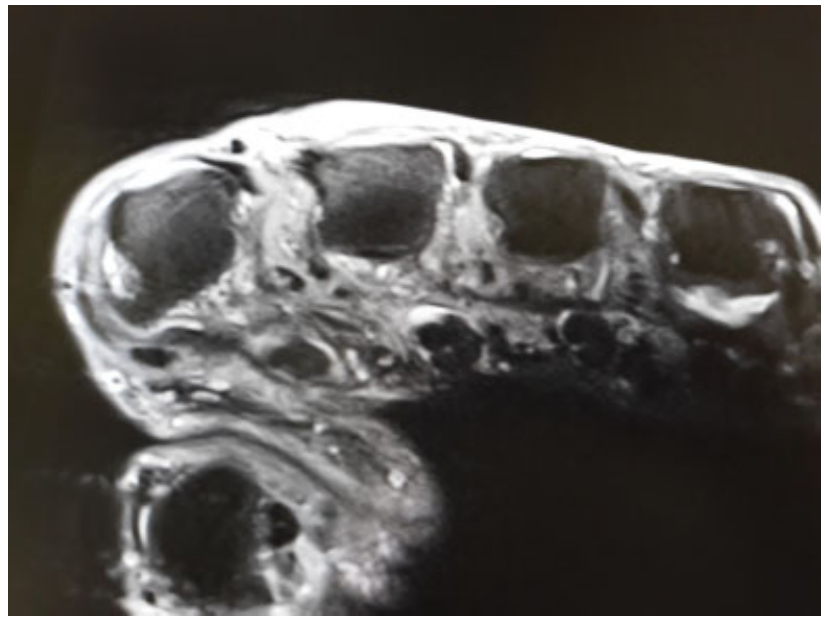

Fig. 13 Imagen de resonancia magnética potenciada en $\mathrm{T} 1$ a nivel de las cabezas de los MTC donde se observan los tendones extensores luxados hacia el lado cubital.

\section{Zona 5-6 (-Fig. 12a, fig. 12b y fig. 12c):}

En el momento agudo, una sección tendinosa proximal a las junturas tendinum puede quedar enmascarada por la transmisión de fuerzas a través de esas estructuras [Tubiana]; pero pasado un tiempo ellas van cediendo y se pierde progresivamente la función extensora del dedo afecto. Para evitar que pase desapercibida una lesión como esa, hay signos clínicos indirectos que pueden indicar una sección del tendón extensor que son el dolor durante la extensión activa del dedo y una fuerza extensora insuficiente. Pero la mejor manera de diagnosticar una lesión tendinosa, es la visión directa del tendón

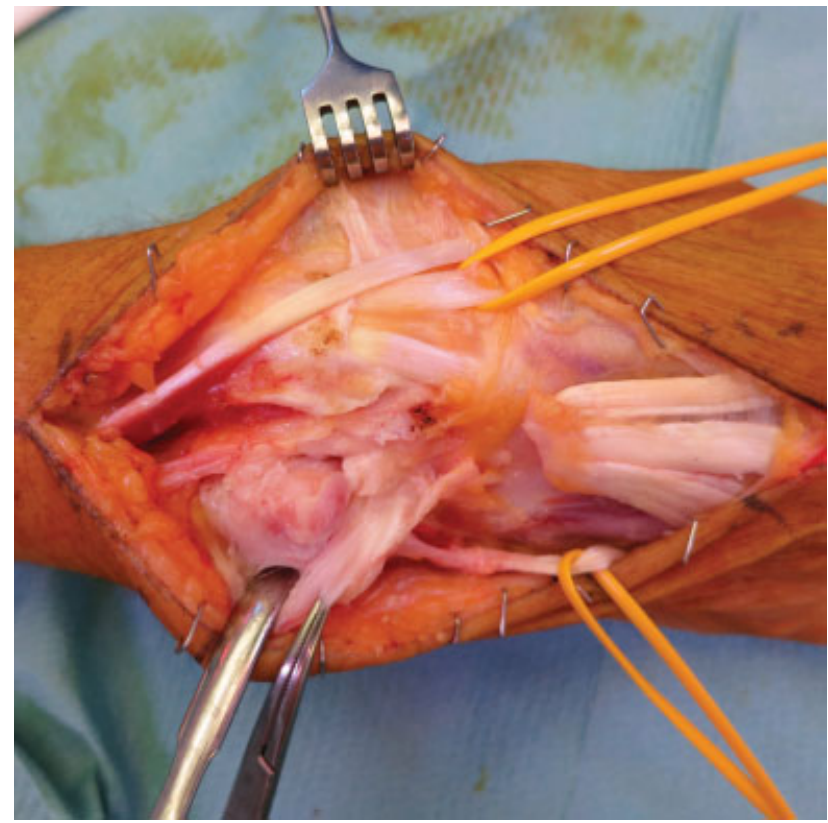

Fig. 14 Imagen quirúrgica de un paciente con un Sd. de Vaughan Jackson, con ruptura de los tendones del ED y el tendón EDM.

seccionado explorándolo a través de la pequeña herida mientras el paciente flexiona y extiende el dedo. De esa manera, pueden apreciarse lesiones del tendón que podrían haber quedado ocultas en caso de no coincidir la herida cutánea con la sección tendinosa por el cambio de relación de esas estructuras en las diferentes posiciones de flexoextensión de los dedos; generalmente si el dedo y la articulación metacarpofalángica están en extensión, la herida cutánea suele coincidir con la sección del tendón extensor y al realizar 
la exploración es más fácil poder objetivar la discontinuidad tendinosa, ya que se suele explorar con los dedos en esa posición. En caso de producirse la herida con las articulaciones metacarpofalángicas en flexión, al estirar los dedos para explorarlos, se producirá un desplazamiento proximal de la zona de sección tendinosa, pudiendo aparentar en la exploración con los dedos en extensión que no ha habido sección tendinosa por estar viendo en realidad, la zona distal del tendón mientras la zona de sección se ha desplazado proximalmente.

Por otro lado, en la sección tendinosa distal a las junturas tendinum, se observa la impotencia funcional para la extensión del dedo desde el inicio dado que hay una disrupción completa del aparato extensor que no permite la transmisión de fuerzas desde la masa muscular extensora hasta la inserción tendinosa el dedo.

También con cierta frecuencia en la zona 5 , en los pacientes reumáticos es característica la luxación de los tendones extensores hacia el lado cubital por la distensión o ruptura de las bandeletas sagitales del lado cubital (-Fig.13).

Zona 7: En la artritis reumatoide se puede producir la ruptura de los tendones extensores por la abrasión sobre la artropatía de la articulación radiocubital distal, conocido con el epónimo de Vaughan Jackson (-Fig.14).
Conflictos de interés Ninguno.

\section{Bibliografía}

1 Bonola A, Caroli A, Cello L. La Mano. Padova: Piccin Editore; 1981

2 Llusa M, Mir X, Suso S, Golano P. Disposición de los tendones extensores común y propio del $5^{\circ}$ dedo a nivel del dorso de la mano. Rev Esp Cir Mano 1991;41(18):75-76

3 Schenck RR. Variations of the extensor tendons of the fingers: surgical significance. J Bone Joint Surg Am 1964;46:103-110

4 Zancolli E, Cozzi EP. Atlas de anatomía quirúrgica de la mano. Madrid: Editorial Médica Panamericana, S.A.; 1993

5 Tubiana R. Surgical repair of the extensor apparatus of the fingers. Surg Clin North Am 1968;48(05):1015-1031

6 Harris C Jr, Rutledge GL Jr. The functional anatomy of the extensor mechanism of the finger. J Bone Joint Surg Am 1972;54(04): 713-726

7 Carrera A, Forcada P, Llusá M. Tendones extensores. Lesiones agudas y deformidades. En: Martínez Martínez F, Marquina Sola P. Técnicas quirúrgicas en cirugía de la mano. Barcelona: Química Farmacéutica Bayer S.L.; 2010:113-131

8 Baratz ME, Schmidt CC, Hughes TB. Lesiones de los tendones extensores. En: Green's cirugía de la mano. Tomo 1. 5a ed. Madrid: Marbán Libros S.L.; 2007:187-217

9 Tubiana R, Thomine J, Mackin E. Movements of the hand and wrist. En: Examination of the hand and wrist. 2a ed. Londres: Martin Dunitz Ltd.; 1996:40-128 